\title{
Eficiência na Provisão de Educação Pública Municipal: Uma Análise em três Estágios dos Municípios Brasileiros
}

\author{
Flávio de Oliveira Gonçalves \\ Professor - Universidade Federal do Paraná \\ Endereço para contato: Av. Prefeito Lothario Meissner, 632 - Jardim Botânico - Curitiba - PR \\ CEP: 80210-170 - E-mail: f.goncalves@ufpr.br
}

Marco Túlio Aniceto França

Professor - Pontifícia Universidade Católica do Rio Grande do Sul

Endereço para contato: Av. Ipiranga, 6681 - Prédio 50, Partenon - Porto Algre - RS

CEP: $90619-900$ - E-mail: marco.franca@pucrs.br

Recebido em 07 de abril de 2011. Aceito em 23 de maio de 2012.

\section{Resumo}

O artigo busca analisar as características municipais que afetam a eficiência dos municípios brasileiros na gestão educacional, consequência do processo de descentralização educacional na década de 90. As informações foram extraídas da Prova Brasil, Censo Escolar, Finbra e STN para 2005. Empregamos um procedimento em três estágios, cujo primeiro consiste no uso de modelos SBM (slacks based measure) no cálculo da eficiência no uso de insumos discricionários. Em seguida, controlamos pelos insumos não discricionários, resultando em um novo índice de eficiência. Finalmente, aplicamos as misturas finitas para analisar a heterogeneidade existente entre os diversos municípios em relação aos níveis de eficiência da gestão municipal. Os resultados mostram a não uniformidade de impactos demográficos, políticos e de recursos sobre a eficiência da gestão educacional entre os diversos grupos de municípios brasileiros. O aumento de democracia e recursos como o decorrente do Fundef, em geral, aumenta a eficiência da administração escolar municipal.

\section{Palavras-Chave}

eficiência, municípios, educação, modelo de misturas finitas

\begin{abstract}
The article analyses what determines efficiency concerning with educational management in Brazilian municipalities, as a result of decentralization process which happened in 90 s. The pieces of information were extracted from Censo Escolar, Prova Brasil Finbra and STN dataset for the 2005-year. We employ a methodology with three stages of which the first stage consists of using the SBM (slacks based measure) models in the efficiency estimation for the use of the discretionary inputs. In addition to this, non-discretionary inputs were controlled of which the result is a new efficiency index. Finally, we use

- O trabalho foi desenvolvido no âmbito do NAPPE - UFPR (Núcleo de Avaliação de Políticas Públicas Educacionais) e os autores agradecem a CAPES e o CNPq pelo apoio financeiro a pesquisa. Possíveis erros e omissões são da responsabilidade dos autores.
\end{abstract}


the finite mixture models to analyse the heterogeneity among the municipalities. The results showed that there is no uniformity in the demographic and political effects on the efficiency of the quality of education on offer among the diversity of the Brazilian municipalities groups. If there is more democracy and funds such as Fundef, in general, it will increase the efficiency of municipal school administration.

\section{Keywords}

efficiency, municipalities, education, finite mixture model

JEL Classification

$\mathrm{C} 14, \mathrm{H} 21, \mathrm{I21}, \mathrm{I} 28$

\section{Introdução}

No Brasil, a descentralização dos recursos educacionais juntamente à expansão das matrículas teve início a partir de 1996, após as reformas constitucionais que culminaram na criação do FUNDEF (Fundo de Desenvolvimento do Ensino Fundamental e de Valorização do Magistério). O fundo criou incentivos para que ocorresse a transferência (parcial ou total) de estudantes das escolas estaduais para as municipais. Todavia, tais mudanças na opinião de Souza, Leme e Paredes (2009), ocorreram sem coordenação entre as secretarias estaduais e municipais de educação, gerando confusão de políticas pedagógicas e perda de economias de escala.

O processo de descentralização pode resultar em acréscimos de eficiência devido ao maior controle social existente sobre os gestores da política pública. Todavia, os contrários à descentralização afirmam que ocorreriam práticas de clientelismo e de "captura" do bem público pela elite local, isto é, com perdas de eficiência. Portanto, parcela da população que tem baixa articulação política não seria beneficiada.

A contribuição do estudo é analisar os fatores que afetam a eficiência da gestão educacional dos municípios, uma vez que as séries iniciais do ensino fundamental, em geral, foram descentralizadas, levando em consideração as diferentes características municipais. O cálculo da eficiência dar-se-á em dois estágios, sendo que o primeiro passo será a aplicação da metodologia de análise de envelopamento de dados (DEA), porém sem a utilização das medidas radiais tradicionais: CCR (Charnes, Cooper e Rhodes, 1978) ou BCC (Banker, Charnes 
e Cooper, 1984), cuja premissa considera retornos constantes ou variáveis de escala, respectivamente. Será utilizada uma medida aditiva: SBM (Slacks Based Measure), que fornece fácil interpretação econômica, mede o grau de ineficiência presente nos insumos e produtos e não necessita definir a priori o tipo de orientação: insumo ou produto, uma vez que no cálculo da eficiência, ambas as orientações são consideradas simultaneamente.

Em um segundo momento, será descontado do cálculo da eficiência as influências relativas às características do corpo discente (etnia, capital humano e econômico dos pais). De acordo com Ruggiero (1996), as variáveis que não estão sob o controle direto do município, denominadas não discricionárias, têm impactos relevantes sob o resultado final. Os municípios cuja proporção de estudantes de escolas públicas possui pais com níveis elevados de capital humano, provavelmente, terão desempenhos superiores em testes de proficiência em relação àqueles de menor nível de capital humano. Assim, utilizaremos uma nova medida de eficiência que será dada pelo resíduo de uma regressão Tobit, procedimento semelhante ao de Ray (1991).

Finalmente, investigar-se-ão os diferentes impactos das características municipais sobre os diversos níveis de eficiência da gestão educacional dos municípios por intermédio de modelos de misturas finitas (Finite mixture Models - FMM). Nos modelos inseriram-se variáveis políticas (competição eleitoral para o executivo e para o legislativo e rotatividade partidária), de população (densidade populacional), de complementaridade de recursos entre União, Estados e Municípios (Fundef) e de desigualdade de renda (Gini). O uso dessa metodologia permitirá capturar a heterogeneidade do impacto das diversas características municipais sobre os níveis de eficiência.

Cabe destacar que os trabalhos que empregam a DEA, os problemas decorrentes de erros de mensuração, variáveis omitidas e outliers podem tornar as estimações e, posteriormente, as conclusões menos confiáveis. Na solução desse problema, Seaver e Triantis (1995) ou Simar (2003) empregaram métodos manuais de inspeção a fim de aplicar o descarte de outliers, contudo, este se mostra impraticável para o caso de grandes amostras. Dessa forma, Sousa, Cribari-Neto e Stosic (2005) aplicaram métodos de bootstrap e jacknife de reamostragem para reduzir os efeitos provenientes dos outliers. Todavia, o seu descarte ou a redução da sua influência pode conduzir à perda 
de informações importantes a respeito de municípios mais e menos eficientes. As conclusões do trabalho mostram que a eficiência decorrente da descentralização beneficia os municípios que estão em ambientes mais democráticos, isto é, que apresentam maior competição eleitoral e rotatividade partidária para o executivo. A descentralização traz aumentos de eficiência para os municípios com menores e maiores populações, não necessariamente, para o grupo dos mais eficientes. A complementaridade de recursos entre União, Estados e Municípios na forma do Fundef logra impactos positivos sobre a eficiência dos municípios.

O artigo está dividido em cinco seções, incluindo esta introdução. A segunda seção aborda brevemente os argumentos favoráveis e contrários à descentralização. A seção seguinte aborda a metodologia desenvolvida no trabalho para o cálculo da eficiência e a estimação do impacto das características municipais sobre a eficiência municipal, consequência da descentralização. A quarta seção refere-se às bases de dados empregadas no trabalho: FINBRA, STN (Secretaria do Tesouro Nacional), Censo Escolar e Prova Brasil e nos informam as características relacionadas aos insumos e produtos empregados no cálculo do SBM, e as características relativas aos insumos não discricionários e aos municípios. Na quinta seção estimamos e discutimos os resultados e fazemos as considerações finais.

\section{Referencial Teórico}

A descentralização diz respeito às características relacionadas à accountability. Isto é, a proximidade entre a população e os responsáveis pela elaboração das políticas públicas resulta em uma menor assimetria de informação entre esses agentes, consequentemente, as necessidades locais seriam mais facilmente atendidas enquanto que os administradores poderiam ser mais responsabilizados (OATES, 1972).

Contudo, reformas descentralizantes, por si só, não são uma garantia de maior eficiência/eficácia na distribuição e oferta dos recursos. Bardhan e Mookherjee (2005) destacam que políticas descentralizantes podem levar à "captura" dos recursos pela elite local. Nesse cenário, os segmentos que não estão no poder simplesmente não 
teriam as suas necessidades atendidas. ${ }^{1}$ Ademais, Tanzi (1995) e Haggard (1999) afirmam que nos municípios prosperariam características relacionadas ao clientelismo, logo conduzindo à ineficiência devido à baixa capacidade de gerenciamento por parte dos gestores locais.

Como os efeitos da descentralização sobre a provisão de serviços educacionais mostram-se ambíguos, Galiani, Gertler e Schargrodsky (2008) utilizam uma função de produção educacional a fim de mostrar as diferenças de eficiência entre uma gestão centralizada (Estados) e descentralizada (Municípios) para a Argentina. Os entes federados apresentam condições socioeconômicas heterogêneas e não haveria a presença de spillovers. ${ }^{2}$

A gestão local será mais eficiente que a regional na existência das seguintes características: as habilidades do corpo técnico local serem superiores ao regional e a existência de complementaridade entre os recursos regionais e locais. Além desses fatores, os autores destacam que a elite governante pode desejar empreender esforços para elevar a oferta educacional. Porém, na ausência desses investimentos, tornase necessário que a população diretamente beneficiada faça valer o seu direito e cobre por tais recursos.

Todavia, Ferreira (2001) conclui por intermédio de um modelo de gerações sucessivas com altruísmo entre gerações que em sociedades com graus elevados de desigualdade poderia haver subinvestimento em educação caso o sistema político fosse do tipo oligárquico. A baixa qualidade aumentaria o custo de oportunidade de frequentar a escola para aqueles com baixas condições socioeconômicas. Desta forma, a descentralização na provisão de recursos educacionais mostrar-se-ia danosa. Assim, os efeitos da descentralização sobre a provisão de serviços educacionais mostram-se ambíguos, pois seriam afetados por características locais. Esses aspectos podem conduzir a um aumento/redução da eficiência no uso dos recursos educacionais. O foco do trabalho é verificar como os aspectos municipais relativos à desigualdade, demografia, recursos e democracia afetariam o sucesso ou não do processo de descentralização, observado pelos níveis

1 Por exemplo, se a elite local não tem filhos matriculados em escolas públicas, a questão da educação pública pode mostrar-se menos importante em comparação às questões relacionadas à segurança.

2 Oates (1972) e Besley e Coate (1999) consideram que na presença de spillovers, uma gestão educacional centralizada é superior a descentralizada. 
de eficiência na gestão do ensino. Na próxima seção descreveremos a estratégia empírica que se utiliza de uma combinação da metodologia de envelopamento de dados (DEA) com o uso de modelos SBM, o controle por variáveis não discricionárias no processo de oferta de ensino e, finalmente, busca-se testar as hipóteses das relações entre variáveis políticas, distributivas e de escala sobre a eficiência da oferta de ensino.

\section{Estratégia Empírica - Determinantes da Eficiência Municipal na Gestão Educacional em três Estágios}

\subsection{Análise de Envelopamento de Dados (DEA)}

$\mathrm{Na}$ literatura existem metodologias paramétricas (fronteira estocástica) ${ }^{3}$ e não paramétricas (DEA) para o cálculo da eficiência. Embora não exista consenso em torno da melhor metodologia (em parte, isto é devido à falta de comparabilidade entre elas), é importante destacar que a paramétrica apresenta como aspectos positivos a incorporação dos erros de medida e os negativos dizem respeito à consideração de um único produto na análise (COOPER, SEIFORD \& TONE, 2000), além da necessidade de suposição de uma forma funcional da função de produção.

O cálculo da eficiência por intermédio de modelos não paramétricos permite a incorporação de inúmeros produtos, fundamenta-se nos axiomas fracos da teoria econômica e não necessita que a forma funcional seja predeterminada. Entretanto, a análise está limitada à amostra, ignora a presença de ruído nos dados e, ademais, a convergência pode ser lenta. A técnica tem sido aplicada nas mais diversas áreas de estudo como: educação (ensino básico ou superior), sistema de transporte público, bancos entre outros. $\mathrm{Na}$ área educacional, a unidade de análise, em sua grande maioria, se dá sobre as escolas ou os estados/municípios devido à facilidade na obtenção de informações. Na literatura internacional podemos citar os trabalhos de Ruggiero (1998 e 2000) que estimam a eficiência das escolas localizadas em Nova Iorque e Farren (2002) que aplica o estudo para as escolas chilenas.

3 Atualmente existem trabalhos que buscam incorporar multiprodutos nos estudos com fronteira estocástica. Um exemplo pode ser encontrado em Fernandez, Koop e Steel (2000).

Est. Econ., São Paulo, vol. 43, n.2, p. 271-299, abr.-jun. 2013 
Para o Brasil destaca-se o trabalho de Delgado e Machado (2007), que mensura a eficiência das escolas públicas estaduais de Minas Gerais. Ao levar em conta um nível mais agregado de análise como os estados e municípios, Faria e Januzzi (2006) calculam a eficiência dos serviços de saúde e educação para os municípios do Rio de Janeiro. Gasparini e Ramos (2003) mensuram a eficiência e a efetividade do ensino médio ofertado pelos estados. Zoghbi, Rocha, Matos e Arvate (2009) utilizam os dados do SAEB-2003 para mensurar a eficiência e o desempenho dos gastos em educação dos estados em relação aos ensinos fundamental e médio. A eficiência dos serviços ofertados pelos municípios é estudada por Sousa e Ramos (1999) e Sousa, Cribari-Neto e Stosic (2005), sendo que no primeiro a análise restringe-se às regiões Nordeste e Sudeste, enquanto que no segundo incorporam-se todos os municípios. É importante destacar que os trabalhos anteriores não controlam os efeitos decorrentes dos insumos não discricionários e que, porventura, afetam os índices de eficiência.

A metodologia empregada será a DEA, porém com a variante de um modelo aditivo denominado SBM (Slacks Based Measure). O método foi criado por Tone (2001) e incorpora os aspectos positivos dos modelos aditivos, ${ }^{4}$ é invariante em relação à unidade de mensuração de insumos e produtos, permite fácil interpretação econômica e, ademais, nos oferece um escalar que mensura o grau de ineficiência presente nos insumos e produtos. Vale ressaltar que a eficiência mensurada com o SBM sofre a influência do conjunto de referência e é monotonicamente decrescente se houver "folgas" na função objetivo.

No trabalho considerar-se-á uma função de produção educacional $(\mathrm{FPE})^{5}$ que está sob gestão dos municípios. Os municípios, $j$, denominados de DMUs, utilizam a FPE para transformar $m$ insumos $x_{i j}=\left(x_{1 j}+x_{2 j}+\ldots+x_{m j}\right) \in \mathfrak{R}_{+}^{M}$ em $s$ produtos

${ }^{4}$ Os modelos aditivos de acordo com Cooper, Seiford e Tone (2000) possuem o mesmo conjunto de possibilidades de produção dos modelos DEA tradicionais e apresentam a vantagem de não necessitar definir a priori qual o tipo de orientação (insumo e produto), uma vez que as "folgas" (excesso de insumo e escassez de produto) são consideradas simultaneamente na função de produção. Além disso, esses modelos são invariantes na transformação de valores negativos em positivos de insumos e produtos no que tange à solução ótima do cálculo de eficiência de acordo com Ali e Seiford (1990). De acordo com Ferreira e Gomes (2009) os modelos podem apresentar pequena variação de magnitude, dada a escala das variáveis escolhidas. Os autores, porém, mostram que as eficiências relativas são mantidas.

5 A resenha clássica está no trabalho de Hanushek (1986). Outros trabalhos que abordam essa temática são Lee e Barro (1997) e Hanushek, Gomes-Neto e Harbison (1996). 
$y_{k j}=\left(y_{1 j}+y_{2 j}+\ldots+y_{s j}\right) \in \mathfrak{R}_{+}^{S}$. A tecnologia utilizada é homogênea e é representada no conjunto de possibilidades de produção T:

$$
T=\{(x, y) \mid x \geq X \lambda, y \leq Y \lambda, \lambda \geq 0\} ; T \subset R^{M+S}
$$

que corresponde a todas as combinações possíveis de insumos e produtos. É importante destacar, que, de acordo com Tone (2001), a condição de não negatividade dos insumos e produtos pode ser relaxada, uma vez que valores negativos podem ser substituídos por pequenos valores positivos. $\mathrm{O}$ índice de eficiência denominado por, $\rho$, tem uma amplitude entre 0 e 1 e a DMU será considerada eficiente quando $(\rho=1)$.

\subsection{Modelo Tobit de Controle de Variáveis não Discricionárias}

O passo seguinte ao cálculo da eficiência consiste na retirada do efeito decorrente das variáveis não discricionárias. ${ }^{6}$ No processo educacional existem variáveis importantes que afetam o desempenho estudantil e que não estão sob o controle direto do gestor tais como: o nível socioeconômico (capital humano e econômico dos pais), peer effects (capital social), a cor da pele autodeclarada pelo estudante, as habilidades inatas e o esforço, por exemplo. Isto é, o objetivo é isolar o efeito que as variáveis não discricionárias (variáveis familiares e relacionados às características estudantis) exercem sob a gestão municipal de educação e, portanto, afetam indiretamente a eficiência da gestão de insumos discricionários.

A fim de controlar pelos não discricionários, Ray (1991) propõe um método em dois estágios que contorna fragilidades como a condução de várias DMUs à fronteira de eficiência e, ademais, mostra-se mais flexível ao lidar com um número elevado de variáveis de ambiente, pois não há a necessidade de determinar o sinal dos coeficientes.

${ }^{6}$ O trabalho seminal no controle dos efeitos não discricionários sobre a eficiência foi realizado por Banker e Morey (1986) e, posteriormente, Ruggiero (1998). Os autores inserem diretamente no modelo DEA, as variáveis não discricionárias. Embora Ruggiero (1998) demonstre que o método de Banker e Morey distorce a medida de eficiência, uma vez que os retornos de escala devem se restringir apenas às variáveis discricionárias, o modelo de Ruggiero apresenta a fragilidade de conduzir várias DMUs à fronteira de eficiência devido ao aumento no número de variáveis não discricionárias. 
No primeiro estágio estima-se um modelo DEA com as variáveis consideradas discricionárias, enquanto no estágio seguinte estima-se uma regressão de mínimos quadrados ordinários com as variáveis não discricionárias como regressores que afetam diretamente o índice de eficiência calculado pela DEA. Assim, a nova medida de eficiência é dada pelo resíduo da regressão e consequência das hipóteses do $\mathrm{MQO}$, a eficiência tem média zero. Ademais, o resíduo da regressão é a eficiência, depois de descontado pelos fatores não discricionários. Contudo, de acordo com Ruggiero (1998), a fragilidade no método reside em seu segundo estágio, pois é preciso especificar a priori a distribuição do resíduo e, portanto, uma má especificação conduziria a uma medida distorcida de eficiência.

O método utilizado no trabalho foi semelhante ao de Ray (1991) para o cálculo da eficiência. Contudo, no segundo estágio, utilizaram-se regressões Tobit ao invés do MQO devido a variável dependente ser censurada em 1 . O método é uma extensão do Probit e é comumente empregado quando certos valores onde o regredido não é observado. Por exemplo, atribui-se zero (um) para as observações que se encontram abaixo (acima) de certo limite e valores positivos para as observações restantes. Assim, para que o resultado reflita a eficiência da gestão municipal, cujos valores não estejam relacionados a qualquer variável não discricionária, é preciso aplicar um método de correção sobre os resíduos de acordo com Ray (2004), uma vez que os resíduos apresentam valores positivos ou negativos.

O regredido é a eficiência da gestão municipal da educação calculada no DEA-SBM $\left(e f f s b m_{j}\right)$, cujos valores são censurados em 1 e os regressores são as variáveis não discricionárias, a saber: os capitais econômico (cap_eco $)^{7}$ e humano (cap_hum) médios dos municípios

\footnotetext{
7 Para as informações relacionadas ao capital econômico (cap_eco) e humano (cap_hum), utilizou-se a metodologia da análise fatorial. É uma técnica multivariada cujo objetivo é descrever as variáveis originais por meio de um número menor de fatores, e que está relacionada ao conjunto original de variáveis por intermédio de um modelo linear. Os fatores são uma dimensão latente a qual se manifesta de forma redundante em algumas variáveis originais (Johnson \& Wichern, 1998). As questões utilizadas buscaram captar a disponibilidade dos seguintes itens na casa do estudante (respostas de 0 a 4): i) televisão em cores, ii) rádio, iii) quartos para dormir, iv) automóvel/carro. Além desses, incluímos uma variável que visa captar a presença de uma pequena biblioteca em casa cuja resposta varia entre 0 a 3. Isto é, $0=$ não existem livros em casa, $1=$ de um a 20 livros, $2=$ entre 20 a 100 livros e 3 = mais de 100 livros. Além de informações a respeito até que série foi cursada pelos pais: $0=$ analfabeto e séries iniciais do ensino fundamental incompleto; 1 = séries iniciais do ensino fundamental completo e fundamental incompleto; 2 = fundamental completo e médio incompleto; 3 = médio completo e superior incompleto; 5 = superior completo. Mais informações no sítio da ABEP - Associação Brasileira de Empresas de Pesquisa: www.abep.org.
} 
e o percentual de estudantes que se autodeclararam: pardos, negros, índios e amarelos. Todas as variáveis foram construídas por meio das informações fornecidas pelos estudantes submetidos aos exames da Prova Brasil.

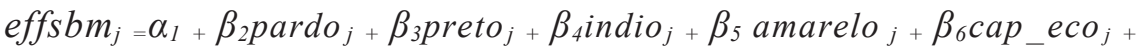
$\beta_{7}$ cap_hum ${ }_{j}+\varepsilon_{j}$

O uso de variáveis familiares justifica-se, na opinião de Bourdieu (1977), devido à estreita relação entre a quantidade de capital humano dos pais e os anos de escolaridade a serem atingidos pela criança, enquanto que o capital econômico garante todo o suporte financeiro para a compra de materiais escolares, acesso à internet, curso de idiomas, entre outros. Além disso, existem diversos trabalhos na literatura nacional e internacional que mostram o impacto positivo das características familiares sobre o desempenho estudantil.

Uma vez estimadas as eficiências municipais, o objetivo deste trabalho é testar as hipóteses levantadas na seção anterior. Não se espera que estas relações sejam homogêneas entre os diversos municípios de nossa amostra. Desta forma propõe-se um modelo de misturas finitas para estimar estas relações.

\subsection{O Modelo de Misturas Finitas (Finite Mixture Models)}

O trabalho seminal que detectou o problema estatístico no tocante às misturas finitas foi Feller (1943). Considere uma população composta de $K$ subgrupos, que estão combinados aleatoriamente e proporcionalmente ao tamanho relativo de cada grupo $\eta_{1}, \ldots, \eta_{k}$. Verifica-se que algumas características aleatórias, $Y$, podem se mostrar heterogêneas entre grupos, contudo, semelhantes intragrupos. Devido à heterogeneidade, $Y$ tem uma distribuição de probabilidade diferente em cada grupo, embora assuma, geralmente, derivar-se de uma mesma distribuição paramétrica, $p(y \mid \theta)$, com o parâmetro $\theta$ diferindo entre os grupos. A variável discreta $S$ denomina os grupos que assume os valores no conjunto $\{1, . ., \mathrm{K}\}$. 
Assim como a variável $Y$, os diferentes grupos, $S$, também podem ser extraídos de uma amostra aleatória cuja probabilidade de amostragem é $\eta_{s}$. Se $S$ é conhecido, $Y$ seguirá uma distribuição $p\left(y \mid \theta_{s}\right), \operatorname{com} \theta_{s}$ sendo o parâmetro no grupo $S$. Logo, a densidade conjunta $p(y, S)$ é dada por:

$$
p(y, S)=p(y \mid S) p(S)=p\left(y \mid \theta_{s}\right) \eta_{s}
$$

Todavia, ao contrário da variável $Y$, os grupos dificilmente são observados. A distribuição de probabilidade dessa variável caracterizase por sua função de densidade probabilidade. Seja $f_{\pi}$, a densidade de distribuição da eficiência da gestão educacional dos municípios, a multimodalidade surge de $f_{\pi}$ como uma mistura finita de outras densidades unimodais, logo:

$$
f_{\pi}=\left(\pi_{\text {mun }}\right)=\eta_{1} h_{1}\left(\pi_{\text {mun }} ; \mu_{1}, \sigma_{1}\right)+\ldots+\eta_{k} h_{k}\left(\pi_{\text {mun }} ; \mu_{k}, \sigma_{k}\right) ; \pi_{\text {mun }}>0
$$

onde $h_{k}\left(\pi_{\text {mun }}\right)$ uma função densidade probabilidade para todo $k=1, \ldots, K$. Uma única densidade, $h_{k}\left(\pi_{\text {mun }}\right)$, refere-se à densidade da componente, enquanto $K$ é o número de componentes ou grupos. Os parâmetros $\eta_{1}, \ldots, \eta_{k}$ são os pesos da distribuição, sendo que os pesos são positivos, $\eta_{i}>0$ e $\Sigma \eta_{i}=1$.

O modelo de misturas finitas consegue lidar com a heterogeneidade não observada e presente em $Y$, uma vez que a distribuição condicional da variável é uma mistura de duas ou mais componentes com médias e variâncias diferentes. O estimador de máxima verossimilhança dá flexibilidade no ajuste aos dados, permitindo que os parâmetros da regressão variem de acordo com a componente e, além disso, a metodologia é capaz de explicar os fatores da heterogeneidade, a partir dos diferentes coeficientes para os parâmetros associados a cada componente.

No processo de especificação é preciso determinar o número de componentes, $K$, uma vez que há uma relação estreita entre o número de componentes e o ajuste aos dados. Todavia, a tarefa ${ }^{8}$ não é

\footnotetext{
8 O teste de razão de verossimilhança, embora seja largamente empregado em misturas finitas, necessita de adaptações no teste devido à violação das condições de regularidade exigidas. Para mais informações veja os trabalhos de Aitkin e Rubin (1985) e Chen, Chen e Kalbfleisch (2004).
} 
das mais simples, uma vez que problemas relacionados à overfitting ${ }^{9}$ tornam os parâmetros não identificáveis. Os critérios para diagnóstico do número de componentes são a razão de verossimilhança (LR), os critérios de Akaike (AIC) e o Bayesiano (BIC) sendo que os dois últimos penalizam modelos mais complexos. Leroux (1992) afirma que o uso desses critérios não implica a subestimação do número verdadeiro de componentes.

\section{A Fonte dos Dados}

As informações municipais utilizadas no trabalho foram extraídas do Censo Escolar, da Prova Brasil, do FINBRA (Finanças do Brasil) e do STN (Secretaria do Tesouro Nacional), respectivamente, para o ano de 2005. O Censo Escolar é o mais importante instrumento de coleta de informações da educação básica e consiste em um levantamento de dados realizado em âmbito nacional e, em todos os anos, por intermédio da colaboração das secretarias municipais e estaduais de educação. A base de dados traz informações sobre as escolas e suas características (corpo docente e infraestrutura física) segundo o município, a dependência administrativa (estadual, municipal, federal e privada), o nível de ensino (educação infantil, ensino fundamental, médio, educação especial e educação de jovens e adultos (EJA), o número de matriculados, concluintes, reprovados e evadidos (movimento e rendimento escolar). Ademais, o Censo 2005 trouxe informações a respeito da presença de diversos programas federais, estaduais e municipais na escola.

Já a Prova Brasil é um exame universal, aplicado somente nas escolas urbanas do sistema público de ensino e que possui corpo discente superior a 20 estudantes. As séries de referência submetidas ao exame são as quartas e oitavas séries do ensino fundamental. A metodologia de avaliação emprega as matrizes de referência que permitem aos alunos responderem a diferentes testes, porém, possuindo itens comuns. O desempenho do estudante é mensurado de acordo com a teoria de resposta ao item, comumente chamada de TRI. Esta variável é o indicador das habilidades e competências dos estudantes,

9 Um potencial overfitting ocorre se uma componente está vazia ou se mais de uma componente possui os mesmos parâmetros. Nesse arcabouço, o modelo pode ser descrito por um número menor de componentes. Este problema está relacionado a não identificação, e pode ser evitado ao restringir que os pesos, $h_{k}$, sejam iguais a zero e que as componentes dos parâmetros sejam diferentes.

Est. Econ., São Paulo, vol. 43, n.2, p. 271-299, abr.-jun. 2013 
ou seja, o indicador de qualidade na sua formação. Os resultados da Prova Brasil vêm acompanhados de informações a respeito das características familiares (cor, sexo, e variáveis socioeconômicas) dos estudantes e a proficiência dos estudantes corresponde a escalas específicas cujos resultados variam de 0 a 500 e propõem-se a avaliar as habilidades e conhecimentos dos estudantes.

Para o primeiro estágio de estimação, quase a totalidade das informações no tocante aos insumos e produtos foram retiradas do Censo Escolar. Logo, a partir de dados primários, construíram-se as seguintes variáveis que entraram na função de produção educacional: a razão entre o número de professores e de estudantes; a razão entre o número de salas de aula e de estudantes; o percentual de professores com ensino superior em relação ao total de professores que lecionam nas séries iniciais, índice de equipamentos, ${ }^{10}$ índice de infraestrutura escolar e um índice ${ }^{11}$ de programas governamentais recebidos pelos municípios. Ao conjunto de insumos incorporou-se a razão entre o PIB e o número de estudantes matriculados nas escolas municipais nas séries iniciais, sendo que a variável foi elaborada com base nas informações do FINBRA para o ano de 2005.

As variáveis que compõem o produto foram três sendo as notas médias: em língua portuguesa e matemática, a taxa de aprovação média e o tamanho da rede municipal presente em cada município.

${ }^{10}$ As variáveis de Equipamentos (infra_equip) e Infraestrutura Física (infra_fisica) foram construídas empregando análise fatorial. A técnica visa identificar os fatores subjacentes que explicam os padrões de correlação dentro de um conjunto de variáveis observadas. É importante ressaltar que as diversas variáveis são resumidas em um único fator por meio da extração do componente principal cujo objetivo será a mensuração da qualidade da infraestrutura de equipamentos e física do município. Para a variável Equipamentos consideraram-se as seguintes quantidades presentes na escola: videocassete; aparelho de TV; retroprojetor; aparelho de fax; maquina copiadora; ventilador nas salas de aulas; mimeografo a álcool, maquina fotográfica. Para a Infraestrutura Física considerou-se a existência das seguintes dependências: laboratório de informática; laboratório de ciências; outros tipos de laboratórios; salas dos professores; diretoria; quadra de esportes (coberta ou descoberta); biblioteca; videoteca; auditório; sanitário (dentro ou fora da escola).

${ }^{11} \mathrm{O}$ índice de programas governamentais (prog_gov) existente no município foi construído utilizando a média aritmética das respostas presentes no questionário do Censo Escolar. É importante destacar que, primeiro, o índice foi elaborado por escola e, posteriormente, agregado pela média municipal. As perguntas cujas respostas $(\operatorname{sim}=1$ e não $=0)$ são: A escola participa do programa Renda Mínima; A escola participa - Programa Nacional TV Escola; A escola participa - Outros programas de TV Educativa; A escola participa - Programa PROINFOMEC; A escola participa - Outros Programas de Informática Educativa; A escola participa - Outros Programas Estaduais; A escola participa - Outros Programas Municipais; A escola participa - Outros Programas Federais; A escola participa - Projeto de Promoção à Saúde Sexual e Reprodutiva; A escola participa - Programa Conferência Nacional Infanto-Juvenil pelo Meio Ambiente. 
Enquanto que as notas médias ${ }^{12}$ da rede municipal são oriundas da Prova Brasil, as informações restantes são fornecidas pelo Censo Escolar. As duas primeiras variáveis compõem o IDEB, e o emprego do número de escolas sob gerência municipal visa controlar a heterogeneidade de escala existente entre os municípios.

Tabela 1 - Estatísticas descritivas - Insumos e Produtos para o cálculo da eficiência

\begin{tabular}{|c|c|c|c|c|c|}
\hline Insumos & Descrição & $\mathrm{N}$ & Média & Min. & Max. \\
\hline Prof_aluno & $\begin{array}{l}\text { Razão entre o número de professores que lecionam } \\
\text { nas séries iniciais e o número de estudantes do } \\
\text { município. }\end{array}$ & 4350 & $\begin{array}{l}0,07 \\
(0,027)\end{array}$ & 0,02 & 0,27 \\
\hline Prof_sup & $\begin{array}{l}\text { Razão entre o número de professores com ensino } \\
\text { superior e o total que leciona nas séries iniciais. }\end{array}$ & 4350 & $\begin{array}{c}0,32 \\
(0,269)\end{array}$ & 0,00 & 1,00 \\
\hline Sala_aluno & $\begin{array}{l}\text { Razão entre o número salas de aula e o número de } \\
\text { estudantes. }\end{array}$ & 4350 & $\begin{array}{c}0,021 \\
(0,014)\end{array}$ & 0,00 & 0,141 \\
\hline Prog_gov & Índice de programas governamentais. & 4350 & $\begin{array}{c}0,26 \\
(0,122)\end{array}$ & 0,00 & 1,00 \\
\hline Infra_fisica & Infraestrutura física da rede municipal. & 4350 & $\begin{array}{c}0,81 \\
(0,814)\end{array}$ & 0,01 & 5,59 \\
\hline Infra_equip & Equipamentos presentes na rede municipal. & 4350 & $\begin{array}{c}0,95 \\
(0,752)\end{array}$ & 0.01 & 6,93 \\
\hline PIB_aluno & $\begin{array}{l}\text { Razão entre o PIB e o número total de estudantes } \\
\text { nas escolas municipais (reais). }\end{array}$ & 4345 & $\begin{array}{l}73.803,68 \\
2.598,018)\end{array}$ & $3.436,15$ & 4.131 .344 \\
\hline Produtos & Descrição. & $\mathrm{N}$ & Média & Min. & Max. \\
\hline Nota & $\begin{array}{l}\text { Média conjunta das disciplinas de língua portuguesa } \\
\text { e de matemática do município. }\end{array}$ & 4350 & $\begin{array}{c}172,11 \\
(16,918)\end{array}$ & 126,38 & 247,81 \\
\hline Taxa_aprov & Taxa de aprovação média do município (\%). & 4345 & $\begin{array}{c}78,69 \\
(12,516)\end{array}$ & 16,00 & 100,00 \\
\hline Num_escolas & Número de escolas da rede municipal. & 4345 & $\begin{array}{c}4,41 \\
(16,250)\end{array}$ & 1,00 & 731,00 \\
\hline
\end{tabular}

Fonte: Elaboração própria a partir do Censo Escolar e Prova Brasil 2005. Desvios padrão entre parênteses.

A Tabela 1 mostra as variáveis discricionárias, isto é, os insumos que estão sob controle direto do município. O índice (prog_gov) que mede a intervenção de outras esferas de governo (Estados e União)

${ }^{12}$ Os desempenhos em exames de proficiência são comumente empregados na literatura DEA de educação para a mensuração do produto, embora exista o reconhecimento de que esses exames não captem características relacionadas à criatividade ou à atitude. 
nas escolas municipais é uma forma de captar o grau de discricionariedade que está nas mãos do prefeito ou do diretor, uma vez que eles são os principais responsáveis pela adoção ou não da política.

As estatísticas descritivas dos insumos não discricionários são mostradas na Tabela 2. As variáveis não discricionárias foram agregadas pela média da escola e, posteriormente, pela média do município. Assim, a eficiência na gestão dos insumos e dos produtos discricionários serão controlados pelas características ligadas ao corpo discente (cor da pele, ${ }^{13}$ capital econômico ${ }^{14}$ e humano familiar). Na Tabela 2, abaixo, as informações são apresentadas:

Tabela 2 - Estatísticas descritivas - Insumos não discricionários

\begin{tabular}{|c|c|c|c|c|c|}
\hline Variáveis & Descrição & N & Média & Min. & Max \\
\hline Pardo & $\begin{array}{l}\text { Percentual de estudantes que se autodeclararam mestiços ( } 1=\text { pardo, } \\
0=\text { outros). }\end{array}$ & 4272 & $\begin{array}{c}0,46 \\
(0,15)\end{array}$ & 0,00 & 1,00 \\
\hline Preto & $\begin{array}{l}\text { Percentual de estudantes que se autodeclararam afrodescendentes ( } 1= \\
\text { negro, } 0=\text { outros). }\end{array}$ & 4272 & $\begin{array}{c}0,09 \\
(0,06)\end{array}$ & 0,00 & 1,00 \\
\hline Índio & $\begin{array}{l}\text { Percentual de estudantes que se autodeclararam descendentes de } \\
\text { indígenas ( } 1=\text { índio, } 0=\text { outros). }\end{array}$ & 4272 & $\begin{array}{c}0,04 \\
(0,04)\end{array}$ & 0,00 & 1,00 \\
\hline Amarelo & $\begin{array}{l}\text { Percentual de estudantes que se autodeclararam descendentes de } \\
\text { asiáticos ( } 1=\text { amarelo, } 0=\text { outros }) \text {. }\end{array}$ & 4272 & $\begin{array}{c}0,03 \\
(0,03)\end{array}$ & 0,00 & 1,00 \\
\hline Cap_eco & Capital econômico familiar médio do município. & 4272 & $\begin{array}{l}-0,27 \\
(0,48)\end{array}$ & $-1,66$ & 1,22 \\
\hline Cap_hum & Capital humano familiar médio do município. & 4272 & $\begin{array}{l}-0,15 \\
(0,25)\end{array}$ & $-1,32$ & 2,17 \\
\hline
\end{tabular}

Fonte: Elaboração própria a partir da Prova Brasil 2005. Desvios padrão entre parênteses.

A Tabela 3 corresponde às estatísticas descritivas dos fatores que podem afetar os diferentes níveis de eficiência dos municípios. As variáveis escolhidas dizem respeito às características político-partidárias, de recursos, populacionais e de desigualdade como: competição eleitoral, ${ }^{15}$ rotatividade partidária (o número de vezes que o partido ascendeu ao poder nas eleições majoritárias entre 1996 e 2004),

${ }^{13}$ As informações no tocante à cor da pele são binárias.

14 Albernaz, Ferreira e Franco (2002) mostram que os estudantes afrodescendentes têm desempenhos inferiores aos seus pares de outras etnias. Contudo, estudantes cujas famílias possuem backgrounds econômicos mais privilegiados apresentam desempenhos superiores em testes de proficiência. Nesse arcabouço, os diferentes níveis de eficiência municipal são indiretamente afetados pelas características dos estudantes que estão matriculados.

15 A competição eleitoral é mensurada pela razão entre o número de candidatos que pleiteiam a vaga e o total de vagas disponíveis. 
tamanho da população, Fundef (Fundo para o Desenvolvimento do Ensino Fundamental e Valorização do Magistério) e desigualdade de renda que é mensurado pelo coeficiente de Gini.

Para a elaboração das variáveis político-partidárias foram empregados os dados do TSE referentes às eleições municipais ocorridas em 2004 e as utilizaremos como indicadores de democracia, pois se espera que elas afetem a eficiência educacional. Embora em um contexto democrático as eleições ocorram sob um ambiente de incerteza até a realização do pleito, é sabido que existe certa fragilidade no uso dessas variáveis para o caso brasileiro, devido à pequena identificação dos partidos com os seus eleitores, embora as suas ações estejam respaldadas no voto popular.

Tabela 3 - Estatísticas descritivas - Variáveis populacionais e político-partidárias

\begin{tabular}{|c|c|c|c|c|c|}
\hline Variáveis & Descrição & $\mathrm{N}$ & Média & Min. & Max. \\
\hline Comp_pref & Competição eleitoral para o cargo de prefeito. & 4349 & $\begin{array}{c}2,93 \\
(1,15)\end{array}$ & 1 & 14 \\
\hline Comp_veread & Competição eleitoral para as câmaras municipais. & 4349 & $\begin{array}{c}6,82 \\
(4,00)\end{array}$ & 1 & 25,88 \\
\hline Rotat_part & $\begin{array}{l}\text { O número de vezes que um mesmo partido ascendeu ao } \\
\text { poder nas eleições majoritárias entre } 1996 \text { e } 2004 .\end{array}$ & 4329 & $\begin{array}{l}2,06 \\
(0,83)\end{array}$ & 0 & 3 \\
\hline Pop & Tamanho da população. & 4348 & $\begin{array}{c}38760 \\
(21882,6)\end{array}$ & 1026 & 10.900 .000 \\
\hline Gini 2000 & Índice de Gini municipal relativo ao ano de 2000. & 4330 & $\begin{array}{c}0,56 \\
(0,57)\end{array}$ & 0,36 & 0,82 \\
\hline Fundef & $\begin{array}{l}\text { Volume de recursos oriundos do Fundef recebidos pelos } \\
\text { municípios (em mil reais). }\end{array}$ & 4349 & $\begin{array}{l}3802,44 \\
(18900)\end{array}$ & 0 & $928.000,00$ \\
\hline
\end{tabular}

Fonte: Elaboração própria a partir da Prova Brasil 2005. Desvios padrão entre parênteses.

As variáveis de competição eleitoral e de rotatividade partidária para o executivo mensuram quão confortável a elite local está no poder. Espera-se que o sinal positivo para ambas as variáveis mostre que existe uma competição eleitoral mais acirrada e que esta seja acompanhada de maiores avanços na eficiência da política educacional, devido à cobrança de parcelas da população que não estão no poder. A competição eleitoral para o legislativo busca captar a diversidade de representantes presentes na população. Esta é responsável pela fiscalização das políticas. Já o índice de Gini será empregado a fim de investigar se os graus elevados de desigualdade de renda reduzem os incentivos para o investimento educacional por parte dos gestores locais. 
Espera-se que quando as diferenças de renda entre o eleitor mediano e o restante da população não se mostrem demasiadamente elevada, de acordo com Saint-Paul e Verdier (1993) e Ferreira (2001), haverá incentivos para que ocorram investimentos educacionais.

Galiani, Gertler e Schargrodsky (2008) destacam que uma das razões para que os resultados decorrentes de um processo de descentralização educacional sejam superiores ao centralizado seja a existência da complementaridade entre fundos estaduais e municipais. Assim, empregamos os recursos do Fundef como forma de mensurar o impacto dessa complementaridade sobre a eficiência, uma vez que é um fundo de âmbito estadual, porém formado com recursos provenientes de todos os entes federados (Municípios, Estados e União) e, cuja divisão ocorre de acordo com o número de matriculados no ensino fundamental sob a sua dependência administrativa. Esta informação foi retirada do STN (Secretaria do Tesouro Nacional) para o ano de 2005 e espera-se que o seu impacto seja positivo sobre a eficiência. A descentralização apresenta impactos positivos quando aproveita os efeitos de escala. Estes estão relacionados à oferta de serviços educacionais e serão medidos pelo tamanho da população. Espera-se que os menores municípios sejam menos eficientes, uma vez que a descentralização estimula a presença de retornos crescentes de escala.

Observa-se que existem municípios que não recebem recursos do Fundef, ou seja, não municipalizaram esse nível de ensino. No geral, observa-se que os municípios brasileiros apresentam características muito diferentes, e isso deve refletir em diferentes impactos sob a eficiência na gestão educacional do município. Na próxima seção, estimar-se-á o nível de eficiência da gestão educacional municipal e, posteriormente, utilizar-se-á da metodologia de misturas finitas (FMM) para investigar o impacto das diferentes características locais (desigualdade de renda, recursos, densidade populacional e democracia) sobre os níveis de eficiência dos municípios. 


\section{Resultados}

A eficiência da gestão municipal nas séries iniciais do ensino fundamental foi calculada para 4350 municípios. De acordo com os resultados do DEA-SBM $\left(e f f_{s} b m_{j}\right)^{16}$ que diz respeito ao primeiro estágio de estimação, observa-se que 294 municípios mostraram-se eficientes, sendo Minas Gerais o estado com o maior número, 57, seguido da Bahia com 49. Verifica-se que os estados, em sua grande maioria, têm um número pequeno de municípios eficientes e os estados de Mato Grosso e Mato Grosso do Sul não possuem um único município eficiente.

Todavia, essa eficiência é afetada por variáveis que não estão sob a gestão do prefeito. Dessa forma, o passo seguinte consistiu na retirada do efeito das variáveis não discricionárias por intermédio da estimação de um modelo Tobit, ${ }^{17}$ cujos regressores foram o nível de capital humano e material familiar médio do município e o percentual de estudantes que se autodeclararam preto, pardo, amarelo e índio. Embora de acordo com a metodologia de Ray (1991), os resíduos da regressão correspondam à eficiência da gestão educacional dos municípios $\left(\pi_{m u n}\right)$, Ray (2004) argumenta que é necessário aplicar um método de correção sobre os resíduos, $\varepsilon_{j}$, uma vez que eles são positivos ou negativos, a fim de se obter uma medida de eficiência gerencial. Portanto, aplicou-se o método de correção de Greene, cujo procedimento visa subtrair de todos os resíduos o maior valor positivo. Como os valores do resíduo tornam-se negativos por default, a aplicação do antilog sobre o resultado passará a refletir a eficiência da gestão municipal, e não estarão relacionados a qualquer variável não discricionária.

Os resultados para a eficiência, após descontar os efeitos dos não discricionários, são apresentados na Tabela 4 segundo a região e, como esperado, observa-se que os municípios da região Sul apresentam uma maior homogeneidade no que tange à eficiência na administração educacional e, ademais, o município de referência (eficiência igual a 1) encontra-se nessa região.

${ }^{16}$ É importante destacar que 36 municípios apresentaram níveis negativos de eficiência. Ao contrário dos modelos DEA tradicionais, o SBM permite a estimação de coeficientes de eficiência negativos. As unidades são denominadas ineficientes e, ao contrário das medidas DEA radiais, ela não exige reduções nos insumos para considerá-las.

${ }^{17}$ Cabe destacar que o emprego do Tobit e a posterior correção de Greene reduzirá o número de municípios que estão na fronteira. Antes da estimação, 294 observações estão censuradas no limite superior, isto é, são iguais a 1 e correspondem a 6,88\% da amostra. 
A Sudeste e a Norte possuem municípios cujos graus de eficiência são bastante heterogêneos, todavia, é importante ressaltar que o município menos eficiente encontra-se na região Sudeste. A CentroOeste detém, na média, a menor eficiência, embora seja uma região com graus de homogeneidade semelhantes a Sul. Cabe destacar que as regiões com as eficiências médias mais elevadas são a Norte e a Nordeste, respectivamente. O resultado mostra-se semelhante ao de Sousa, Cribari-Neto e Stosic (2005) para a oferta geral de serviços públicos.

Tabela 4 - Estatísticas da eficiência após controlar os efeitos não discricionários segundo a região

\begin{tabular}{l|c|c|c|c|c}
\hline Região & $\mathbf{N}$ & Média & Desv. Padrão & Min. & Max \\
\hline Norte & 352 & 0,42 & 0,15 & 0,08 & 0,86 \\
Nordeste & 1504 & 0,41 & 0,13 & 0,02 & 0,84 \\
Sudeste & 1256 & 0,39 & 0,15 & 0,0002 & 0,95 \\
Sul & 794 & 0,37 & 0,09 & 0,21 & 1 \\
Centro-oeste & 365 & 0,34 & 0,09 & 0,08 & 0,81 \\
\hline
\end{tabular}

Fonte: Elaboração própria.

Ao investigar a eficiência educacional na administração municipal das capitais vis-à-vis ao IDEB, observa-se que existe uma fraca correlação $(2,89 \%)$ entre eficiência e eficácia (IDEB) dos municípios. De acordo com a Tabela 5 abaixo, enquanto Salvador é a capital com menor eficácia, uma vez que detém o pior desempenho no IDEB, é a terceira mais eficiente. Conclui-se que a diferença nos resultados mostra que a prefeitura de Salvador possa empregar adequadamente os insumos, mas o baixo background estudantil pode comprometer o produto além da possibilidade de uma ocorrência de subinvestimento. A situação não é exclusiva de Salvador, pois outras capitais nordestinas (Recife e Teresina) apresentam cenários semelhantes. Na região Sudeste, São Paulo seria a capital mais eficiente, embora seja a sétima no ranking do IDEB. A capital que alia eficácia e eficiência é o Rio de Janeiro, pois tem a segunda maior eficiência e a terceira maior eficácia. 
Tabela 5 - Ranking segundo a Eficiência e o IDEB (Índice de Desenvolvimento do Ensino Básico)

\begin{tabular}{l|c|c|c|c|l|l|l|l|l}
\hline Capitais & Efic. & $\begin{array}{l}\text { Efic. } \\
\text { Rank. }\end{array}$ & IDEB & Rank. & Capitais & Efic. & $\begin{array}{l}\text { Efic. } \\
\text { Rank. }\end{array}$ & IDEB & Rank. \\
\hline São Paulo & 0.874 & 1 & 4.1 & 7 & Boa vista & 0.411 & 14 & 3.9 & 11 \\
Rio de Janeiro & 0.847 & 2 & 4.2 & 3 & Macapá & 0.407 & 15 & 3.4 & 18 \\
Salvador & 0.716 & 3 & 2.8 & 26 & Campo Grande & 0.392 & 16 & 4.2 & 5 \\
Porto velho & 0.543 & 4 & 3.5 & 16 & Maceió & 0.375 & 17 & 3.1 & 22 \\
Manaus & 0.542 & 5 & 3.5 & 17 & Florianópolis & 0.369 & 18 & 4.2 & 6 \\
Curitiba & 0.534 & 6 & 4.7 & 1 & João pessoa & 0.368 & 19 & 2.9 & 24 \\
Recife & 0.504 & 7 & 3.2 & 20 & Natal & 0.364 & 20 & 3.3 & 19 \\
Teresina & 0.495 & 8 & 4.2 & 4 & Belém & 0.360 & 21 & 3 & 23 \\
Belo Horizonte & 0.493 & 9 & 4.6 & 2 & Porto alegre & 0.356 & 22 & 3.6 & 15 \\
Goiânia & 0.443 & 10 & 3.9 & 10 & Rio branco & 0.354 & 23 & 4.1 & 8 \\
Fortaleza & 0.432 & 11 & 3.2 & 21 & Vitória & 0.353 & 24 & 4.1 & 9 \\
São Luís & 0.416 & 12 & 3.6 & 14 & Palmas & 0.319 & 25 & 3.8 & 12 \\
Cuiabá & 0.415 & 13 & 3.7 & 13 & Aracaju & 0.317 & 26 & 2.9 & 25 \\
\hline
\end{tabular}

Fonte: Elaboração própria.

As capitais da região Sul notabilizam-se pela baixa eficiência, à exceção de Curitiba, embora com níveis elevados de eficácia. Isto seria um indicador de um uso não adequado dos recursos. Já Aracaju e Belém estariam "no pior dos mundos", pois se mostram menos eficientes e, ademais, possuem o segundo e o quinto menores desempenhos no IDEB. Pode-se afirmar que os municípios mais eficazes na provisão dos serviços educacionais não são sempre os mais eficientes. Os resultados são semelhantes aos encontrados para os estados por Zoghbi, Rocha, Matos e Arvate (2009). 


\subsection{Definição dos Grupos de Eficiência de Gestão Educacional dos Municípios}

A fim de verificar a existência de mais de uma componente $(K>1)$, aplicou-se a metodologia de misturas finitas em praticamente ${ }^{18}$ todos os municípios, cujas escolas estão sob a administração municipal. $\mathrm{Na}$ Tabela 6, abaixo, apresentam-se as estimativas do teste $L R$ modificado para a comparação de modelos de uma, duas, três, quatro e cinco componentes. Os critérios de seleção para a rejeição do modelo será com base no valor $p$ e na estatística de qui-quadrado. Ademais, empregar-se-ão os critérios de $A I C$ e BIC que mensuram a qualidade do ajustamento do modelo aos dados.

Tabela 6 - Critérios de AIC e BIC e Teste Modificado de Razão de Verossimilhança (LR)

\begin{tabular}{ccccc}
\hline Componentes & AIC & BIC & Componentes & Teste LR (valor $p$ ) \\
\hline Um & $-5201,35$ & $-5188,63$ & - & - \\
Dois & $-9755,43$ & $-9723,63$ & Modelo 1 vs 2 & 0,000 \\
Três & $-10005,08$ & $-9954,2$ & Modelo 2 vs 3 & 0,000 \\
Quatro & $-10016,2$ & $-9946,242$ & Modelo 3 vs 4 & 0,007 \\
Cinco & $-10013,08$ & $-9924,05$ & Modelo 4 vs 5 & 0,41 \\
\hline
\end{tabular}

Fonte: Elaboração própria.

Os resultados mostram a presença de multimodalidade no índice de eficiência educacional da gestão municipal, sugerindo que não devemos analisar os aspectos que influenciam os diferentes níveis de eficiência considerando uma única distribuição. Os critérios de AIC e $B I C$ nos conduziriam a uma possível ambiguidade, pois de acordo com o AIC, o modelo de quatro componentes mostra-se superior aos modelos com três ou cinco componentes, contudo, o resultado não é semelhante ao BIC. É importante destacar que o resultado é decorrência do AIC favorecer modelos mais complexos, na opinião de Bozdogan (1987). O BIC, de acordo com Keribin (2000), mostra-se mais consistente e sugere especificações parcimoniosas, uma vez que cada um dos componentes fornece um ajuste adequado aos dados.

${ }^{18}$ Os municípios submetidos ao exame da prova Brasil totalizaram 4.350. Contudo, devido às informações faltantes em algumas variáveis não discricionárias, foram avaliados $4271 \mathrm{mu}$ nicípios por intermédio do modelo de misturas finitas. 
O teste modificado de razão de verossimilhança, juntamente com o AIC, mostram que o modelo com quatro componentes é preferível em relação aos demais ao nível de significância de $1 \%$.

A Tabela 7 (anexo) apresenta as estatísticas descritivas para as variáveis participantes da função de produção educacional sob gestão municipal e que entraram no cálculo do DEA-SBM. Além dessas, a tabela também mostra as estatísticas descritivas das variáveis políticas (competição para o executivo, o legislativo e rotatividade partidária), populacionais (tamanho da população), desigualdade de renda (Gini) e o volume de recursos (Fundef) e que entraram na estimação do modelo de misturas finitas. Os municípios são agrupados nas componentes de acordo com os níveis de eficiência constituindo uma relação crescente (do menos eficiente para o mais eficiente) e aplicou-se um teste de diferenças de médias a fim mostrar a presença de heterogeneidade entre as variáveis.

A primeira componente encontra os municípios menos eficientes, sendo composto por 2178 municípios. A eficiência desse grupo tem amplitude entre um valor bem próximo de zero e 0,35. Esta componente possui, na média, coeficientes inferiores em relação às demais no que tange a todas as variáveis, isto é, competição eleitoral para os cargos de prefeito e de vereador, alternância de partidos no poder executivo, tamanho da população e volume de recursos oriundos do Fundef. É importante destacar, é a componente cujos prefeitos permitem maior intervenção governamental em suas escolas, provenientes de outras esferas administrativas, além de maior percentual de professores com ensino superior. O teste de médias mostra que os coeficientes dessa componente são estatisticamente diferentes da componente 2, que é formado pelos municípios um pouco menos ineficientes.

A segunda componente concentra 1110 municípios cuja eficiência na gestão educacional está entre 0,35 e 0,41 . As diferenças são estatisticamente significantes em relação à terceira componente para as variáveis relativas à desigualdade de renda, competição para o executivo e recursos do Fundef. A terceira componente é formada por 469 municípios que possuem, na média, o maior contingente populacional. Os níveis de eficiência têm amplitude entre 0,41 e 0,52 e apresentam como características uma elevada competitividade eleitoral tanto para o executivo quanto para o legislativo, assim 
como alta alternância de partidos no poder. A quarta componente reúne os 512 municípios mais eficientes. As variáveis de competição eleitoral para o legislativo e para o executivo apresentam coeficientes menos elevados quando comparados à componente anterior, embora as variáveis sejam estatisticamente diferentes. Por fim, não existem diferenças significativas em relação aos recursos do Fundef entre as componentes 3 e 4, contudo, são nos municípios mais eficientes que, em média, recebem os valores mais elevados.

No modelo seguinte, as variáveis político-partidárias serão empregadas como regressores segundo o tipo de eleição (majoritária e proporcional). É importante destacar que as políticas educacionais são decididas no âmbito do executivo, embora a fiscalização da política, além da representação das mais diversas correntes da sociedade, espera-se que esteja no legislativo. A eficiência da gestão educacional municipal, $\pi_{m u n}$, de acordo com Oates (1972), é uma das consequências do processo de descentralização, e este será estimado tendo como regressores as variáveis de competição eleitoral para o executivo, para o legislativo além da rotatividade partidária. Todas essas variáveis serão utilizadas como indicadores do grau de pressão exercido pela população e cujas políticas a beneficiam diretamente, como destacado por Galiani, Gertler e Schargrodsky (2008). Os mesmos autores destacam a importância do fundo que complementa os recursos provenientes dos diversos entes federados e, para isso, utilizaremos os recursos do Fundef. O tamanho da população é indicador escolhido para captar economias de escala, além de utilizarmos o índice de desigualdade de renda (Gini) como destacado por Ferreira (2001). A Tabela 8 sumariza os resultados:

Tabela 8 - Estimação do Modelo de Misturas Finitas

\begin{tabular}{l|c|c|c|c}
\hline \multicolumn{1}{c|}{ Variável dependente: eficiência } & Componente 1 & Componente 2 & Componente 3 & Componente 4 \\
\hline Competição para o executivo & -0.001 & $-0.016^{\star}$ & 0.0006 & $0.009^{\star \star \star}$ \\
Competição para o legislativo & $0.02^{\star \star \star}$ & 0.005 & 0.0001 & -0.001 \\
Rotatividade partidária & $0.004^{\star}$ & 0.011 & 0.0001 & $0.007^{\star \star}$ \\
Tamanho da população & $7.82 \mathrm{e}-08^{\star \star \star}$ & $-7.25 \mathrm{e}-08$ & $1.09 \mathrm{e}-07$ & $-5.91 \mathrm{e}-08$ \\
Grau de desigualdade & 0.061 & -0.008 & $-0.17^{\star \star \star}$ & 0.06 \\
Fundef & $1.71 \mathrm{e}-09^{\star \star \star}$ & $-1.05 \mathrm{e}-10$ & $7.65 \mathrm{e}-09^{\star \star \star}$ & $1.15 \mathrm{e}-09^{\star \star \star}$ \\
Constante & $0.268^{\star \star \star}$ & $0.584^{\star \star \star}$ & $0.405^{\star \star \star}$ & $0.312^{\star \star \star}$ \\
Proporção da amostra & $25,76 \%$ & $19,44 \%$ & $33,71 \%$ & $21,09 \%$ \\
\hline
\end{tabular}

Fonte: Elaboração própria.

*** $1 \%$, ** $5 \% \mathrm{e}^{*} 10 \%$ 
Em geral, as variáveis são estatisticamente significativas a $5 \%$ e os sinais das variáveis mostram que existem diferenças no impacto sobre a eficiência de acordo com a componente em que se encontra o município. O sinal da variável de competitividade eleitoral para o executivo mostra-se positivo e estatisticamente significante para os municípios mais eficientes. Por outro lado, o sinal mostra-se negativo e estatisticamente significante para os grupos de municípios menos eficientes. É um indicativo de que a descentralização não beneficiou igualmente todos os municípios, pois disputas acirradas pelo poder beneficiaram apenas os mais eficientes como destacam Galiani, Gertler e Schargrodsky (2008). A variável de rotatividade partidária mostra-se positiva e estatisticamente significante para os municípios que estão nas caudas da distribuição. Nas demais, o resultado não foi estatisticamente significante. O resultado corrobora o observado na competição para o legislativo, pois somente os menos eficientes que apresentam ganhos de eficiência. Parece existir uma evidência de que nos municípios menos eficientes a descentralização não gerou uma suficiente responsabilização que gerasse aumentos nos níveis de eficiência na gestão municipal.

A economia de escala mensurada pelo tamanho da população mostra um impacto positivo e significante sobre a eficiência municipal somente para a primeira componente. Logo, as economias de escala beneficiariam os níveis de eficiência daqueles grupos de municípios composto pelos menores municípios. A desigualdade de renda mostra-se, em geral, estatisticamente não significante à exceção para a terceira componente, sendo que a variável tem impacto negativo sobre a eficiência. Por fim, é importante destacar que a complementaridade entre os recursos provenientes da União, Estados e Municípios e que forma o Fundef gera acréscimos de eficiência na gestão educacional, à exceção do observado na segunda componente. O resultado está de acordo com uma das razões para que uma gestão descentralizada seja melhor que a centralizada, na opinião de Galiani, Gertler e Schargrodsky (2008). 


\section{Conclusão}

O artigo investiga como os diferentes níveis de eficiência municipal na gestão educacional, ocasionados pela descentralização do ensino a partir da adoção do Fundef, são afetados por diferentes características municipais. Para isso, inicialmente utilizou-se de uma função de produção educacional para calcular os diferentes níveis de eficiência. A metodologia empregada foi a SBM (Slacks Based Measure), uma variante dos modelos DEA aditivos. O método incorpora os aspectos positivos dos modelos DEA tradicionais, além de permitir a estimação dos níveis de eficiência sem a necessidade da determinação do tipo de orientação. Na etapa seguinte, empregamos um modelo Tobit a fim de controlar o efeito que as variáveis não discricionárias exercem sobre a eficiência. Ao não isolar este efeito, a tendência é que ocorra uma superestimação da eficiência dos municípios com ambientes mais favoráveis. Os resíduos da regressão fornecem os novos parâmetros de eficiência da gestão educacional municipal após aplicar a correção de Greene. Os resultados mostram que há uma baixa correlação entre eficácia e eficiência, e que muitas capitais consideradas pouco eficazes apresentam níveis de eficiência elevados no uso dos recursos.

O modelo de misturas finitas foi empregado para explicar os determinantes dos diferentes níveis de eficiência. A metodologia permite mensurar parâmetros diferentes segundo a componente, portanto, consegue captar as diversas fontes de heterogeneidade. Os resultados do modelo para quatro componentes, de acordo com os critérios de $A I C$ e de razão de verossimilhança, mostram que a competitividade para o executivo municipal beneficia somente o grupo de municípios mais eficientes. Outra variável relativa à democracia e que mede a permanência do partido no poder, mostra que o impacto sobre a eficiência se dá nas caudas da distribuição. Já a competitividade no legislativo apenas beneficia o grupo de municípios menos eficientes. Portanto, um dos objetivos da descentralização, que é o aumento da transparência no uso dos recursos com impactos positivos sobre a eficiência, logra resultados positivos desde que aplicados em ambientes mais democráticos.

O tamanho da população mensura se a descentralização beneficia os municípios maiores devido à presença de economias de escala. A descentralização beneficiou não somente os municípios mais po- 
pulosos, mas também aqueles com menor população. Haveria um efeito no formato de $U$ invertido em que os municípios localizados nas caudas da distribuição da variável de tamanho da população se beneficiariam em maior proporção dos efeitos de escala, contudo, este impacto não beneficia os municípios mais eficientes.

Os recursos oriundos do Fundef contribuíram no acréscimo de eficiência sendo uma evidência de que a descentralização educacional na gestão tem que vir acompanhada de aporte de recursos dos outros entes federados a fim de auxiliar os municípios. A descentralização dos serviços educacionais conduz a aumentos nos níveis de eficiência, embora o impacto não se mostre semelhante para todos os municípios. O resultado pode ser consequência da baixa capacidade municipal em realizar política educacional e, em alguns municípios, a descentralização não estaria sendo eficaz na redução das assimetrias de informação entre a comunidade local e o gestor. Em alguns casos, mostrar-se-ia interessante uma maior intervenção governamental via mecanismos de responsabilização política, além de um maior aporte de recursos.

\section{Referências}

AITKIN, M. \& RUBIN, D. B.: Estimation and hypothesis testing in finite mixture models. Journal of the Royal Statistical Society Series B (Statistical Methodology). v. 47, n. 1. pp. 67-75. 1985.

ALBERNAZ, A., FERREIRA, F. \& FRANCO, C. (2002): Qualidade e eqüidade na educação fundamental brasileira. Pesquisa e Planejamento Econômico, v. 32, n.3, dez. 2002.

ALI, A. I. \& SEIFORD, L. M.: Translation invariance in data envelopment analysis. Operations Research Letters, 9, pp. 403-405. 1990.

BANKER, R. D., CHARNES, A. \& COOPER, W. W.: Some models for estimating technical and scale inefficiencies in data envelopment analysis. Management Science. v. 30, n.9 pp.1370-1382. 1984.

BANKER, R.D. \& MOREY, R.: Efficiency analysis for exogenously fixed inputs and outputs. Operations Research. v. 34, pp. 513-521. 1986.

BARDHAN, P. \& MOOKHERJEE, D. Decentralizing antipoverty program delivery in developing countries. Journal of public economics. n.89.v.4.p.675-704. 2005.

BOURDIEU, P. Cultural Reproduction and Social Reproduction. In: Power and Ideology in Education, edited by Jerome Karabel and A. H. Halsey, New York: Oxford University Press, 1977.

BOZDOGAN, H.: Model selection and Akaike's information criterion (AIC): The general theory and its analytical extensions. Psychometrika n. 52, n.3. sept. pp. 345-370. 1987.

CHARNES, A; COOPER, W.W.; RHODES, E.: Measuring the efficiency of decision making units. European Journal of Operational Research, v. 2, n. 6, p. 429-444. 1978.

CHEN. H., CHEN, J. \& KALBFLEISCH. J. D.: Testing for a finite mixture model with two components. Journal of the Royal Statistical Society. B (Statistical Methodology). v.66, n.1 pp. 95-115. 2004.

Est. Econ., São Paulo, vol. 43, n.2, p. 271-299, abr.-jun. 2013 
COOPER, W.W., SEIFORD, L.M. \& TONE, K.: Data Envelopment Analysis: A Comprehensive Text with Models, Applications, References and DEA-Solver Software, Kluwer Academic Publishers. Boston. 2000.

DELGADO, V. M. S. \& MACHADO, A. F.: Eficiência das Escolas Públicas Estaduais de Minas Gerais. Pesquisa e Planejamento Econômico. v. 37, pp. 427-464. 2007.

FARIA, A. F.; JANUZZI, P. M.: Eficiência dos gastos municipais em saúde e educação: uma investigação através da análise envoltória no Estado do Rio de Janeiro. Rio de Janeiro: IPEA. (Prêmio: IPEA-CAIXA 2006; Concurso de Monografias. Tema 1: Eficiência e Efetividade do Estado no Brasil). 2006.

FARREN, D. The technical efficiency of schools in Chile. Applied Economics. n.34. pp.1533-1542. 2002.

FELLER, W.: On a general class of contagious distributions. Annals of Mathematical Statistics. v. 14, pp. 389-400. 1943.

FERNANDEZ, C.; KOOP, G. \& STEEL, M. A Bayesian analysis of multiple output production frontiers. Journal of Econometrics, v. 98, pp. 47-79. 2000.

FERREIRA, F. Education for the masses? The interaction between wealth, educational and political inequalities. Economics of Transition, v. 9, n. 2. pp. 533-552. 2001.

FERREIRA, C.M. de C.\& GOMES, A. P. Introdução à análise envoltória de dados: teoria, modelos e aplicações. Editora UFV. Viçosa, 2009.

GALIANI, S.; GERTLER, P. \& SCHARGRODSKY, E.: School decentralization: helping the good get better, but leaving the poor behind. Journal of Public Economics, Elsevier, v. 92, pp. 2106-2120, 2008.

GASPARINI, C. E. \& RAMOS, F. S.: Efetividade e Eficiência no Ensino Médio Brasileiro. Economia Aplicada, v.7 n. 2. pp.389-411. 2003.

HAGGARD, S. The reform of the state in Latin America. World Bank's Annual Conference on Development in Latin America and the Caribbean. Rio de Janeiro, 12-13 June 1995.

HANUSHEK, E. A.; GOMES-NETO, J. B.; HARBISON, R. W.: Efficiency-enhancing investments in school quality. In: BIRDSALL, N. \& SABOT, R. H. (Eds.). Opportunity forgone: education in Brazil. Washington, DC. Inter-American development Bank, pp.385-424. 1996.

HANUSHEK, E. The Economics of Schooling: Production and Efficiency in Public Schools, Journal of Economic Literature. v. 24, n.3, pp.1141-1177. 1986.

JOHNSON, R. \& WICHERN, D.: Applied multivariate statistical analysis. 4ed. New Jersey. Prentice Hall. 1998.

KERIBIN, C. (2000). Consistent estimation of the order of mixture models. Sankhya. The Indian journal of statistics A, v. 62, pp.49-66. 2000.

LEE, J.; BARRO, R. J. Schooling quality in a cross section of countries. Cambridge: NBER, 1997 (Working paper, 6198). Disponível < http://www.nber.org/papers/w6198.pdf?new_window=1> acesso: 08 ago de 2009.

LEROUX, B. G.: Consistent estimation of a mixing distribution. The Annals of Statistics v. 20, pp. 1350-1360. 1992.

OATES, W. E.: Fiscal federalism. Harcourt Brace Javanovich, New York. 1972.

RAY, S. C. Resource use efficiency in public schools. A study of Connecticut data. Management Science. n. 37, pp. 1620-1628. 1991.

RAY, S. C. Data envelopment analysis. Theory and techniques for economics and operations research. Cambridge: Cambridge university press. 376p. 2004.

RUGGIERO, J. On the measurement of technical efficiency in the public sector. European Journal of Operational Research. n.90. pp.553-565. 1996.

RUGGIERO, J.: Non-discretionary inputs in data envelopment analysis. European Journal of Operational Research v. 111, pp. 461-469. 1998.

Est. Econ., São Paulo, vol. 43, n.2, p. 271-299, abr.-jun. 2013 
RUGGIERO, J.: Measuring technical efficiency. European Journal of Operational Research. v. 121, pp. 138-150. 2000.

SAINT-PAUL, G. \& VERDIER, T.: Education, democracy and growth. Journal of Development Economics. v. 42, n.2 pp. 399-407. 1993.

SEAVER, B. \& TRIANTIS, K. The impact of outliers and leverage points for technical efficiency measurement using high breakdown procedures. Management Science. v. 41 n.6, pp.937-956. 1995.

SIMAR, L. Detecting Outliers in Frontier Models: A Simple Approach. Journal of Productivity Analysis, 20, pp. 391-424, 2003.

SOUSA, M. C. S. \& RAMOS, F. S. Eficiência técnica e retornos de escala na produção de serviços públicos municipais: o caso do nordeste e do sudeste brasileiros. Revista Brasileira de Economia. v.4, n.53, p.433-461. out-dez. 1999.

SOUSA, M.C. S., CRIBARI-NETO, F. \& STOSIC, B.D. Explaining DEA technical efficiency scores in an outlier corrected environment: The case of public services in Brazilian municipalities. Brazilian Review of Econometrics, v. 25 pp. 289-315. 2005

SOUZA, A. P. F.; LEME, M. C. \& PAREDES, R.: A municipalização do ensino fundamental e seu impacto sobre a proficiência no Brasil. In: VELOSO, F.; PESSSOA, S.; HENRIQUES, R. \& GIAMBIAGI, F. (Org.). Educação Básica no Brasil. Rio de Janeiro: Campus/Elsevier, 2009.

TANZI, V. Fiscal federalism and decentralization: a review of some efficiency and macroeconomic aspects. Annual Bank Conference on Development Economics. Washington, May 1-2, 1995.

TONE, K.: A slacks-based measure of efficiency in data envelopment analysis. European Journal of Operational Research. v. 130, pp. 498-509. 2001.

ZOGHBI, A. C. P., ROCHA, F. F., MATOS, E. H. C. \& ARVATE, P. R.: Mensurando o desempenho e a eficiência dos gastos estaduais em educação fundamental e média. Estudos Econômicos. v.39. n.4 out-dez. pp.785-809. 2009. 


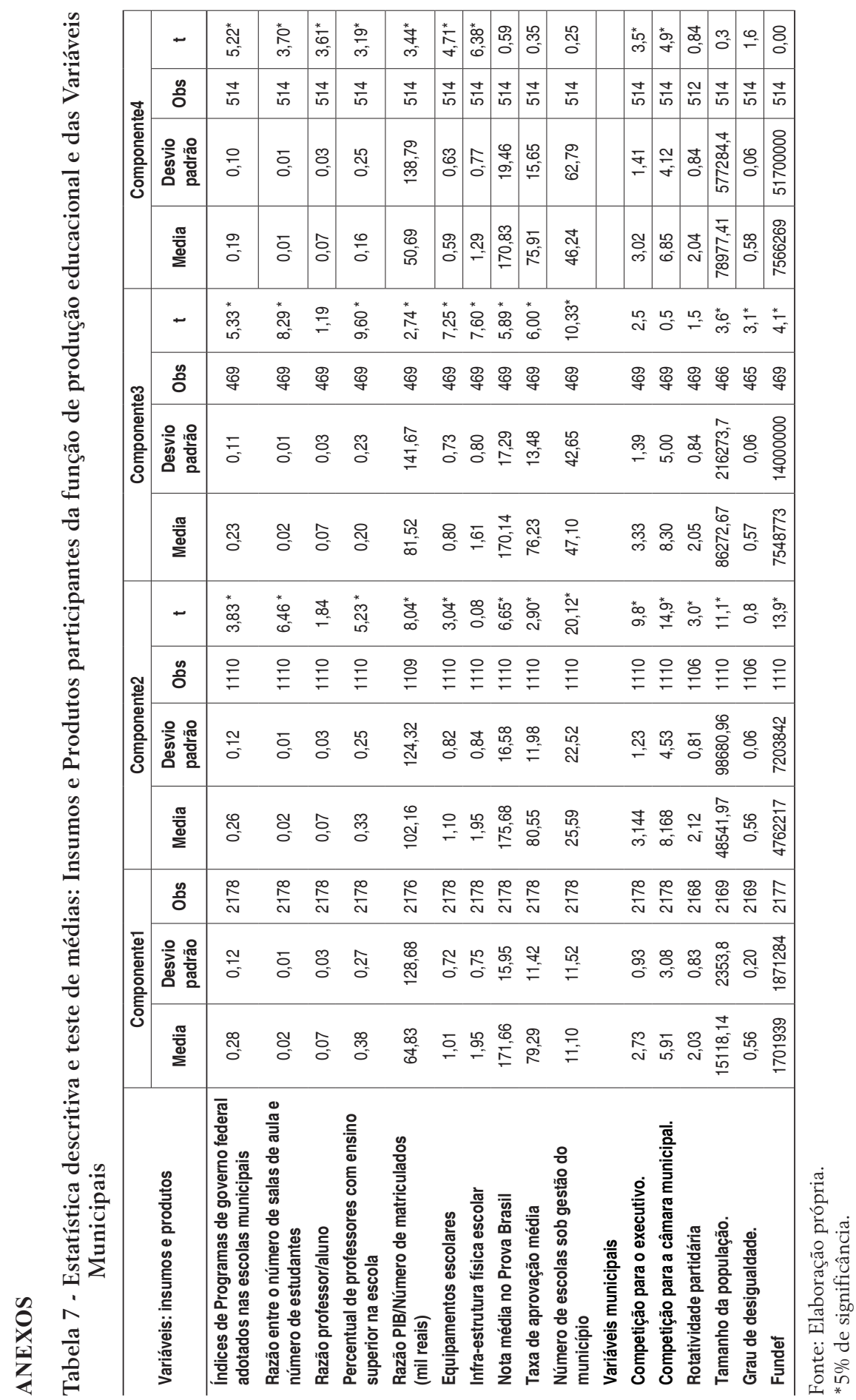

